\title{
Universiteit
}

Leiden

The Netherlands

\section{The effects of experimentally lowered serotonin function on emotional information processing and memory in remitted depressed patients.}

Merens, W.; Booij, L.; Haffmans, P.M.J.; Does, A.J.W. van der

\section{Citation}

Merens, W., Booij, L., Haffmans, P. M. J., \& Does, A. J. W. van der. (2008). The effects of experimentally lowered serotonin function on emotional information processing and memory in remitted depressed patients. Journal Of Psychopharmacology, 22, 653-662. Retrieved from https://hdl.handle.net/1887/14399

Version: $\quad$ Not Applicable (or Unknown)

License: $\quad$ Leiden University Non-exclusive license

Downloaded from: https://hdl.handle.net/1887/14399

Note: To cite this publication please use the final published version (if applicable). 


\section{The effects of experimentally lowered serotonin function on emotional information processing and memory in remitted depressed patients}

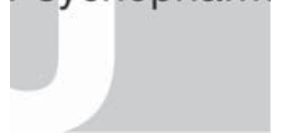

Journal of Psychopharmacology 22(6) (2008) 653-662 (c) 2008 British Association for Psychopharmacology ISSN 0269-8811

SAGE Los Angeles, London, New Delhi and Singapore $10.1177 / 0269881107081531$

W Merens Department of Psychology, Leiden University, Leiden, The Netherlands.

L Booij Department of Psychiatry, McGill University, Montréal, Quebec, Canada.

PM Judith Haffmans Department of Psychology, Leiden University, Leiden, The Netherlands; Psychomedical Center Parnassia, The Hague, The Netherlands.

AJW van der Does Department of Psychology, Leiden University, Leiden, The Netherlands; Department of Psychiatry, Leiden University, Leiden, The Netherlands.

\begin{abstract}
It has been well documented that acute tryptophan depletion (ATD) induces symptoms in remitted depressed patients treated with an SSRI. ATD also has effects on cognition, both in patients and in healthy samples. The exact nature of ATD-induced cognitive changes in depression remains unclear. It is also unclear whether cognitive effects can be induced through partial ('low-dose') depletion. The aim of this study is to investigate the differential effects of low-dose and high-dose ATD on emotional information processing and mood in remitted depressed patients.

Eighteen remitted depressed patients received high-dose and low-dose ATD in a randomized, double-blind, within-subjects crossover design. Mood was assessed before and after administration of the depletion drink. Five hours after administration, patients conducted tests measuring neutral and emotional information processing.
\end{abstract}

High-dose ATD increased depressive symptoms and induced a temporary depressive 'relapse' in half of the patients. High-dose ATD also decreased the recognition of fear and impaired learning and memory retrieval. The impaired learning occurred only in mood-responders. Low-dose ATD had no effects on mood but speeded the recognition of facial expressions of disgust. Accurate recognition of sad faces at baseline was associated with mood response to ATD.

High-dose ATD leads to changes in memory and in the recognition of negative facial expressions in SSRI-treated remitted depressed patients. The effect of low-dose ATD on mood and cognition seems to be quite limited. Emotional information processing at baseline predicts moodresponse to ATD.

\section{Introduction}

Acute tryptophan depletion (ATD) is a useful tool to investigate the effects of lowered serotonin function in humans (Young et al., 1985). With this procedure plasma tryptophan is temporarily reduced by $70-90 \%$. Central serotonin function is also affected (Carpenter et al., 1998; Nishizawa et al., 1997). ATD results in a temporary depressive 'relapse' in $50-60 \%$ of remitted depressed patients taking serotonergic anti-depressants (Van der Does, 2001). In healthy individuals only small mood effects are found in subjects with a family history of affective disorders (Benkelfat et al., 1994; Klaassen et al., 2002).
ATD also has cognitive effects. In healthy volunteers, ATD selectively impairs learning (Park et al., 1994), memory retrieval and consolidation (Klaassen et al., 2002; Park et al., 1994; Riedel et al., 1999) and ATD improves attention in healthy samples (Schmitt et al., 2000) and patients (Booij et al., 2005a). Recently, interest has been paid to the effects of ATD on emotional information processing. ATD impaired the recognition of facial expressions of fear in healthy female volunteers, but not in males (Harmer et al., 2003b).

Three studies investigated the effects of 'low-dose ATD', which involves administering fewer and/or smaller amounts of amino acids (AAs) compared with 'high-dose ATD'. In both healthy and recovered depressed individuals, an increased negative attentional bias was 
found following low-dose ATD, in absence of any effects on mood (Hayward et al., 2005). Also, in recovered depressed patients lowdose ATD reduced the recognition of happiness, increased startle response, impaired initial memory of neutral words (Hayward et al., 2005 ) and increased processing of social threat cues (Munafò et al., 2006). Booij et al. (2005a), however, found only minor effects of lowdose ATD on cognition in remitted depressed patients. In that study, selective attention for neutral stimuli improved following high-dose and low-dose ATD in a dose-dependent manner and the processing of positive information was impaired only following high-dose ATD (Booij et al., 2005a). A limitation of this study is that the cognitive test battery consisted mainly of neutral stimuli, which may explain the differences with Hayward et al. (2005) and Munafò et al. (2006). It is also possible, however, that differences in the low-dose procedures are responsible. The traditional (high-dose) ATD procedure consists of 15 AAs; amounting to $102.5 \mathrm{~g}$. Booij et al. used the Krahn et al. (1996) procedure as their low-dose condition, which consists of a $25 \%$ amount of the same 15 AAs $(25.7 \mathrm{~g})$. Although developed as a placebo, this procedure reduces plasma tryptophan levels by $40-50 \%$ (Van der Does, 2001). Hayward et al. and Munafò et al. however, used eight of these 15 AAs in approximately $50 \%$ amount (a total of $31.2 \mathrm{~g}$ ). Another difference is that in Booij et al. (2005a) patients kept a low-Trp diet the day before the ATD sessions. Calculations using the data provided by Hayward et al. reveals that their low-dose mixture resulted in an $87 \%$ decrease in the ratio of tryptophan to large neutral AAs (LNAA). This effect is similar to the decrease found following high-dose depletion in other studies. The low-dose mixture in Booij et al. (2005a) resulted in a decrease of only $42 \%$ in the tryptophan/LNAA ratio. Hence, it may be doubted whether the studies by Hayward et al. and Munafò et al. may be viewed as low-dose ATD studies (Merens and Van der Does, 2007).

The present study is the first to investigate the effects of lowdose versus high-dose ATD on emotional information processing, and aims to answer three questions:

1. Do low-dose and high-dose ATD have differential effects on emotional information processing and memory in remitted depressed patients?

2. Does ATD exert a specific effect on (a certain form of) emotional information processing or is the effect generalized over different aspects of emotional information processing?

3 . Is there a relationship between emotional information processing and changes in mood?

To investigate this, different cognitive tests were used to assess neutral (memory) as well as emotional information processing (negative attentional bias, facial expression recognition, implicit attitudes) in remitted depressed patients. Two different ATD dosages were used to investigate a possible dose-response relationship. This study extends on previous work by combining two ATD dosages to test the effects on emotional information processing and memory in remitted depressed patients and by investigating the link between emotional information processing and changes in mood. On the basis of previous research we hypothesized that high-dose ATD would increase symptoms in a subgroup of patients, but no mood effects of low-dose were expected (Booij et al., 2005a). We also hypothesized that both high-dose and low-dose ATD would affect attentional bias and facial expression recognition (Harmer et al., 2003b; Hayward et al., 2005; Munafò et al., 2006) and that the cognitive effects of low-dose ATD would be strongest in participants with a depressive response to high-dose ATD.

\section{Methods}

\section{Participants}

Participants were outpatients of the Mood Disorders clinic of a psychiatric hospital. Inclusion criteria were 18-65 years; primary DSM-IV diagnosis of major depressive disorder; no longer fulfilling DSM-IV criteria for depression; Hamilton rating scale for Depression scores below 16; ongoing treatment with a selective serotonin reuptake inhibitor (SSRI) or a selective serotonin-noradrenalin reuptake inhibitor (SSNRI) for at least four weeks; no psychosis (lifetime); no substance abuse in the past three months (DSM-IV criteria); (above) normal weight (BMI > 18); free of neuro-endocrine and neurological disease; no pregnancy or lactation.

\section{Design}

The study was conducted according to a double-blind, randomized crossover design.

Composition of the amino-acid mixtures The high-dose AA mixture consisted of fifteen AAs (102.5 g) (Delgado et al., 1990). The low-dose mixture consisted of the same AAs but at one quarter strength (25.7 g) (Krahn et al., 1996). The AAs were given in drink form by mixing the amino acid powders with water to a final volume of $300 \mathrm{~mL}$. To compensate for the unpleasant taste of the mixture, chocolate syrup was added and the mixtures were served chilled.

\section{Materials}

Symptoms Symptoms were assessed using the Montgomery Asberg depression rating scale (MADRS) (Montgomery and Asberg, 1979) and the brief anxiety scale (BAS) (Tyrer et al., 1984). A self-rating list of physical symptoms was administered, consisting of 48 items rated on a five-point scale. State versions of all questionnaires were used during the depletion sessions. Because changes in weight or sleep do not occur during the course of an ATD session, these items were omitted.

Cognition Facial expression recognition test: This task, adapted from Harmer et al. (2003b), features examples of five basic emotions - happiness, sadness, fear, anger and disgust (Ekman and Friesen, 1976). Emotional expression intensity was morphed between neutral (0\%) and emotional standard (100\%) in 10\% steps, providing a range of emotional intensities. Each emotion-intensity was presented twice (one male and one female face) in random order on a computer screen for $500 \mathrm{~ms}$ and immediately replaced by a blank screen. Participants responded by pressing a labelled key, 
after, which the next face appeared. They were instructed to respond as quickly and accurately as possible. Accuracy and speed of recognition were recorded.

Word learning test (Saan and Deelman, 1986): A list of 15 unrelated, neutral words was presented from a tape (e.g. flower, store). Immediate recall was tested after each of five consecutive presentations. After the fifth trial, subjects continued with a non-verbal task. Fifteen minutes later delayed recall was tested. Different versions were used at each session in a randomized order. Immediate recall performance was defined as the total of correct words remembered over the five trials. Retrieval was defined as the number of correct words produced at delayed recall. Incorrect responses were also registered. Consolidation was defined as the number of correct words at delayed recall compared with the fifth trial.

Verbal fluency: Participants were instructed to produce as many correct four letter words with the same initial letter as possible within one minute. The starting letters were H, M, R or L (Schmitt et al., 2000) and the order of these letters was randomized over the assessments. The total number of correct and incorrect words was calculated.

Dot probe test: This task measures attentional bias to emotional stimuli (MacLeod et al., 1986). Word pairs were presented on a computer screen for $500 \mathrm{~ms}$, one in the upper part of the screen and one below. Following the termination of that display, a dot appeared on the location of either word. Participants had to indicate the location of the dot by pressing a key. All word pairs were preceded by a white fixation cross for $500 \mathrm{~ms}$. Subjects were first run through six practice trials. The main experiment consisted of trials of neutral words paired with threat-related words and trials of depression-related words paired with positive words. The neutral and threat-related words were taken from Lavy, van den Hout and Arnzt (1993) and the depression and positive words from McCabe, Gotlib and Martin (2000). To control for possible outliers, median latencies for correct responses were used in the analyses; incorrect responses and responses on practice trials were excluded. Attentional bias was calculated by subtracting the RT for positive (neutral) words from the RT for depressive (threatening) words. Attentional bias is associated with faster reaction times if the dot replaces the negative word compared with trials where the dot replaces the neutral or positive word.

Implicit association test: The Implicit association test (IAT) is a sorting task that assesses implicit associations on the basis of reaction times (Egloff and Schmukle, 2002; Greenwald et al., 1998). This test is extensively used in social psychological research to assess stereotypes (Greenwald and Banaji, 1995). Participants are asked to sort stimuli representing four categories by pressing the appropriate key (each response key was assigned to two categories). If two categories are strongly related, the sorting task will be easier (: faster RTs) when the categories share the same response key than when they share different response keys. We used an emotional and a neutral version of this task. In the neutral version, negative and positive words were presented, which were paired with flower- and insect words. RTs to congruent (e.g., flower words paired with positive words) and incongruent (e.g., insect words paired with positive words) trials were calculated. The emotional version was identical to the neutral version except for the stimuli: self-related words were paired with negative words and otherrelated words with positive words and vice versa. The negative and positive words were taken from McCabe, Gotlib and Martin (2000); the neutral words were selected by Lavy, van den Hout and Arntz (1993) and the self and other words were taken from Egloff and Schmukle (2002) and were previously used by De Jong (2002). To control for possible outliers, median latencies for correct responses were included in the analyses; incorrect responses were excluded. Reaction times to congruent (e.g. self and positive words) and incongruent stimuli (e.g. self and negative word pairs) were calculated.

Dysfunctional Attitudes Scale (DAS) (Weissman, 1979) is a selfrating scale and assesses the level of dysfunctional attitudes, which is a measure of cognitive reactivity. Two comprised 11-item versions, based on the original form A and were used in randomized order.

Biochemical measures To determine total plasma tryptophan concentrations and the ratio total tryptophan/ LNAA, venous blood was obtained $(10 \mathrm{~mL})$ in ethylene-diamine-tetra-acetic-acid tubes. After sampling, the blood was centrifuged for $20 \mathrm{~min}$ at $2650 \mathrm{~g}_{\max }$. Plasma was stored at $-65^{\circ} \mathrm{C}$ until quantitative AA analysis by high-performance liquid chromatography took place (as described by Fekkes et al., 1995).

\section{Procedure}

After showing interest in taking part, all volunteers were given oral and written information about the study. Written informed consent was obtained and participants who seemed to meet inclusion criteria were invited for a screening session. During the screening session, the Structured Clinical Interview for DSM-IV (First et al., 1995), HAM-D17 and MADRS were administered. Participants filled out the personality questionnaires. A clinical psychologist or a trained research assistant conducted the interviews. At screening the cognitive tasks were done for the first time. The day before each ATD session, participants kept a low-protein diet (Booij et al., 2005a). Participants arrived at 9 AM at the laboratory after fasting overnight. They were instructed not to drink alcohol $24 \mathrm{~h}$ prior to the sessions and to arrive well rested. After arrival, baseline symptom measures were taken, followed by a blood sample. Next, participants ingested the AA mixture within half an hour. Participants remained in the research room until 5 PM. Neutral music and magazines were available. Participants were not allowed to sleep. Water, (de)caffeinated coffee and (herb) tea were allowed in standard amounts. Three hours after ingestion of the AA mixture, participants were served a protein-poor lunch (Booij et al., 2005a; Riedel et al., 1999). At $\mathrm{t}_{4.5}$ the cognitive tasks were administered, which took approximately $50 \mathrm{~min}$. Next, a blood sample was obtained and symptoms and side effects were assessed $\left(t_{6.5}\right)$. Before the participants went home at $5 \mathrm{PM}$, they received a snack to speed up normalization of tryptophan levels. The procedure was repeated approximately seven days later; participants who had first received the $100 \%$ strength mixture now received the $25 \%$ strength mixture and vice versa. The morning after each session, patients returned to the lab where symptoms were assessed and a blood sample was 
obtained. The day after the second ATD session, participants performed the cognitive tests a final time (postintervention session). The study was approved by an independent medical ethics committee (METIGG, Utrecht). Participants were tested individually. Participants were paid $€ 115$, - for participation.

\section{Statistical analysis}

The effects of ATD on symptoms and cognitive performance were analysed separately using general linear models for repeated measures. Symptom ratings were analysed with Intervention (low-dose versus high-dose) and Time (predepletion versus postdepletion versus the next day) as within-subject factors. Cognitive performance was analysed with Intervention (baseline versus low-dose versus high-dose) as within-subject factor. In those instances in which the assumption of homogeneity of covariance in repeated measured analysis was violated, as assessed with Mauchly's test of sphericity, Huynh-Feldt corrected $P$-values were used (Field, 2005). Main effects were compared using Bonferroni corrections to adjust for multiple comparisons. Results are reported as means and standard deviations.

\section{Results}

\section{Data screening}

All data were screened for accuracy of data-entry, missing values and the assumptions of multivariate data-analysis. Data were missing for one patient at the postintervention session following the second day, owing to an emotional reaction to the high-dose depletion drink. After having contacted the patient by telephone the next morning, she reported to be less emotional but she was still very tired and did not want to come to the lab. For the MADRS and BAS, data for this patient were replaced by the outcome of a regression equation based on her mood in the afternoon, because we knew her symptoms had not returned to baseline the next morning (when $\mathrm{X}=\operatorname{mood}$ in the afternoon, $\mathrm{A}=$ constant, $\mathrm{b}=$ unstandardized coefficient and $\mathrm{Y}=$ mood the next morning: $\mathrm{Y}=\mathrm{A}+\mathrm{b} . \mathrm{X})$. The missing biochemical measures were replaced by the sample mean. The MADRS, BAS and side-effect data were log transformed owing to non-normal distributions. All transformations were successful.

\section{Participants}

Twenty-patients were included. One patient withdrew after the screening session owing to family problems. One patient dropped out after the first day (high-dose depletion) owing to physical and mood complaints following that session. These complaints had disappeared the day after, however, she decided to withdraw participation. These patients were excluded from analyses, leaving a total of eighteen participants. Five participants vomited after high-dose ATD; one of them also vomited after low-dose ATD. One patient could only finish $75 \%$ of the drink on both days. All these cases were retained. Clinical and demographical characteristics of the patients are shown in Table 1.
Table 1 Clinical and demographical characteristics $(N=18)$

\begin{tabular}{ll} 
M/F & $2 / 16$ \\
Age (years) & $44.4(13.3)$ \\
Type of medication & $n=12$ \\
$\quad$ SSRI $^{\mathrm{a}}$ & $n=6$ \\
$\quad$ SSNRI $^{\mathrm{b}}$ & \\
Type of remission & $n=7$ \\
$\quad$ Partial remission & $n=11$ \\
$\quad$ Full remission & $13.4(22.9)$ \\
Duration of remission, partial or full (months) & $26.5(15.6)$ \\
Age of onset of first depressive episode & $4.8(4.2)$ [range 1-15] \\
Number of previous episodes (incl. latest) & $2 / 16$ \\
Single / recurrent & $n=12$ \\
Family History of Depression (1st degree) & $25.5(3.4)$ \\
BMI & $7.8(3.7)$ \\
HDRS (at screening) & \\
\hline
\end{tabular}

${ }^{a}$ citalopram $20-60 \mathrm{mg}$, fluoxetine $40 \mathrm{mg}$, paroxetine $10-40 \mathrm{mg}$, sertraline $100 \mathrm{mg}$.

benlafaxine $150-375 \mathrm{mg}$.

Values represent means with SD in parentheses.

SSRI, selective serotonin reuptake inhibitor; SSNRI, selective serotonin and noradrenalin reuptake inhibitor; BMI, body mass index; HDRS, Hamilton depression rating scale.

\section{Effects on plasma tryptophan levels}

In the low-dose condition, mean plasma tryptophan levels decreased with $60.3 \pm 13.3 \%$ from $42.8 \pm 5.1$ to $17.3 \pm 6.9 \mu \mathrm{mol} / \mathrm{L}$. In the high-dose condition, plasma tryptophan levels decreased with $84.0 \pm 11.0 \%$ from $41.9 \pm 7.1$ to $6.7 \pm 4.4 \mu \mathrm{mol} / \mathrm{L}$. The plasma tryptophan/LNAA ratio decreased with $58.5 \pm 15.9$ in the low-dose condition (from $10.4 \pm 1.6$ to $4.3 \pm 1.6$ ) and with $91.4 \pm 8.7$ in the high-dose condition (from $10.0 \pm 1.6$ to $0.82 \pm 0.80$ ). Repeated measures analysis on the tryptophan/LNAA ratio revealed significant effects of Intervention $(F(1,17)=59.7, P<0.001)$, Time $(F(1.64,27.81)=266.0, P<0.001)$ and Intervention $X$ Time $(F(2,34)=28.9, P<0.001)$. Both interventions resulted in a significant decrease in plasma tryptophan and the tryptophan/ LNAA ratio and the decrease was larger after high-dose than after low-dose ATD. Since a sizable number of patients had vomited following the high-dose AA mixture, all analyses were run twice, both with and without those five patients. All effects regarding the plasma tryptophan and tryptophan/LNAA ratio remained significant with 13 patients.

\section{Side effects}

Physical complaints increased in the high-dose but not in the lowdose condition and levels were back to normal the next morning (Intervention $F(1,16)=5.0, P=0.039$; Time $F(2,32)=17.0$, $P<0.001$; Intervention $\times$ Time $(F(2,32)=5.7, P=0.008)$. The main effects remained significant for $N=13$, however, the interaction effect became a trend $(F(2,22)=2.9, P=0.079)$. Significant Intervention $\times$ Time interactions were found for individual items 
indicating a decreased appetite $(F(1.28,20.41)=8.1, P=0.006)$, increased nausea $(F(2,32)=14.4, P<0.001)$ and sweaty hands $(F(2,32)=4.1, P=0.027)$. Symptoms were back to baseline the next morning.

\section{Effects on symptoms}

Depressive symptoms increased significantly in the high-dose condition from $5.3 \pm 5.4$ to $10.8 \pm 8.1$, but not in the low-dose condition (from $3.7 \pm 4.0$ to $3.6 \pm 4.2$ ). All scores were back to baseline the next morning (Intervention $F(1,17)=17.3$, $P=0.001$; Time $F(2,34)=4.6, P=0.018$; Intervention $\times$ Time $F(2,34)=3.9, P=0.030)$. High-dose ATD induced a brief 'depressive relapse', defined as an increase in MADRS score of six points or more (Booij et al., 2005a) in nine patients. Relapses occurred in four of twelve SSRI users and five of six SSNRI users, indicating mood response to be equally distributed over SSNRI and SSRI users. These patients will be referred to as mood-responders. When relevant, analyses were re-run including Mood-response as a between-subject factor. Anxiety symptoms also increased following high-dose ATD but not following low-dose ATD (Intervention $F(1,17)=2.9, P=0.105$, Time $F(2,34)=3.2, P=0.053$, Intervention $\times$ Time: $F(2,34)=4.6, P=0.017)$. The effects of ATD on symptoms remained the same when the mood data were re-analysed, excluding the five patients who vomited. Of the five patients who vomited, two were mood-responders.

\section{Learning effects, baseline measures and order effects}

To detect possible learning effects, screening and postintervention assessments were compared for all cognitive tests. No learning effects were found on the Dot-probe and Word learning test. On the VF, patients produced more correct words postintervention compared with screening $(t(16)=-4.5, P<0.001)$. On the IAT neutral, patients were faster to respond to congruent $(t(16)=2.6$, $P=0.021)$ as well as incongruent stimuli $(t(16)=4.8, P<0.001)$ at postintervention compared with screening. This was also true for the IAT emotional (congruent stimuli $t(16)=4.1, P=0.001$; incongruent stimuli $t(16)=2.8, P=0.012$ ). Patients were better at recognizing sadness $(t(16)=2.8, P=0.012)$, anger $(t(16)=4.3$, $P=0.001)$, disgust $(t(16)=2.4, P=0.029)$ and fear $(t(16)=3.4$, $P=0.004)$ at the postintervention session compared with screening. Patients were also faster in recognizing the different emotions at postintervention compared with screening $(P$ values ranging from 0.000 to 0.051 ). To control for these effects, a baseline cognitive performance score was calculated by taking the mean score of the screening session and the postintervention session (the day after the second depletion session). This procedure is in lieu of a baseline measure at the morning of each ATD session (cf. Booij et al., 2005a). Repeated measures analyses were done with Intervention (baseline versus low-dose versus high-dose) as within-subject factor.

Effects of Order of administration (low-dose ATD first versus high-dose ATD first) were investigated by including Order as a between subjects factor in the analyses. Only on the IAT emotional, an interaction effect of Intervention $\times$ Order was found for incongruent stimuli $(F(2,32)=5.7, P=0.007)$ indicating that patients who received the low-dose first, became faster to respond to incongruent stimuli following both low-dose and high-dose. Patients who received the high-dose first became slower to respond to incongruent stimuli following high-dose ATD.

\section{Effects on neutral information processing}

Word learning task Immediate recall: An Intervention effect was found for the number of correct $(F(2,34)=4.1, P=0.026)$ and incorrect $(F(1.24,21.07)=6.0, P=0.018)$ responses. Post hoc contrast tests indicated fewer correct responses after high-dose depletion compared with baseline $(F(1,17)=4,9, P=0.040)$ and low-dose depletion $(F(1,17)=5.4, P=0.033)$. Also, more incorrect responses were given following high-dose depletion compared with baseline (main effect of Intervention $F(1,17)=8.2$, $P=0.033$ ) and low-dose ATD (a trend for an Intervention effect: $F(1,17)=3.9, P=0.066)$.

Retrieval: An Intervention effect was found for the number of correct $(F(1.45,25.58)=9.8, P=0.002)$ and incorrect $(F(1.56,26.58)=4.2, P=0.035)$ words at $+15 \mathrm{~min}$. Contrast tests revealed that the number of correct words decreased after high-dose ATD compared with baseline $(F(1,17)=14.5$, $P=0.001)$ and low-dose depletion $(F(1,17)=8.4, P=0.010)$. The number of incorrect responses increased following high-dose compared to baseline (effect of Intervention $F(1,17)=5.6$, $P=0.031)$ and low-dose (a trend of an Intervention effect $F(1,17)=4.3, P=0.055)$.

Consolidation: Main effects of Intervention $(F \quad(1.29$, $21.99)=6.7, P=0.012)$ and Time $(F(1,17)=21.2, P<0.001)$ were found. The interaction effect of Time $\times$ Intervention was not significant $(P>0.1)$. Subjects recalled fewer words at delayed recall compared with immediate recall (fifth trial), but there were no differences between the conditions. When data were re-analysed with $N=13$, results were equal.

Verbal Fluency: The number of correct and incorrect words were analysed separately with Intervention as a within-subjects factor. No effects of high-dose and low-dose depletion were found on any of the verbal fluency measures $(P>0.10)$.

\section{Effects on emotional information processing}

Facial emotion recognition test Accuracy: Overall accuracy data were analysed with Intervention and Emotion as within-subject factors. Subjects were better at recognizing certain emotions compared with others (main effect of Emotion $F(4,68)=24.1, P<0.001$ ) and they performed differently over the interventions (main effect of Intervention $F(2,34)=4.1, P=0.026$ ) (see Figure 1$)$. When re-analysed with $N=13$, the Intervention effect became a trend $(F(2,24)=3.0$, $P=0.071)$ but the effect of Emotion remained significant $(P<0.001)$. Separate analyses were done for each emotion with Intervention and Intensity (in five $20 \%$ blocks) as within-subject factors. Decreased fear recognition was found following high-dose ATD (Intervention $F$ (2, $34)=4.1, P=0.025$; Intensity $F(3.12,52.96)=233.1, P<0.001$; Intervention x Intensity $F(8,136)=2.0, P=0.052)$ as compared with low-dose $(F(1,17)=6.2, P=0.023)$ and baseline $(F(1,17)=6.3$, $P=0.023)$. The Intervention effect was significant for the $30-40 \%$ 


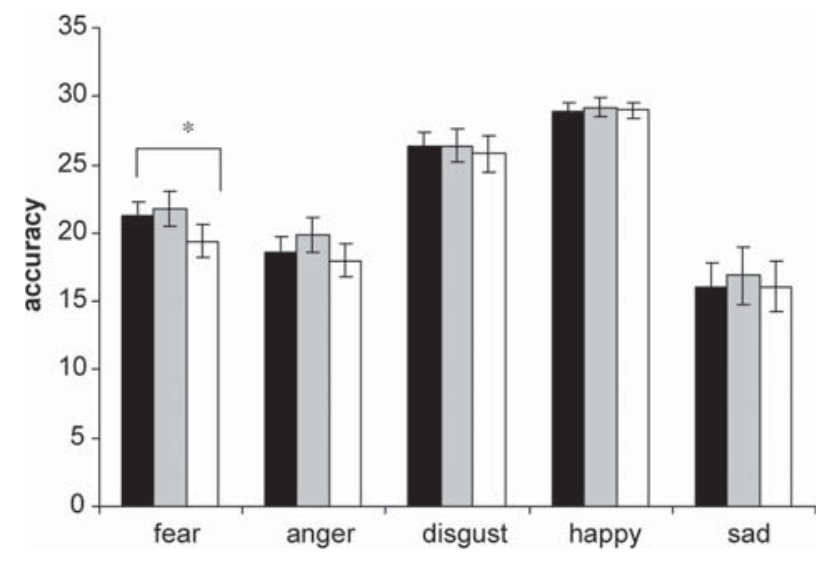

Figure 1 Facial expression recognition at baseline (dark bars), following low-dose depletion (grey bars) and high-dose depletion (white bars) Values represent means \pm 1 SEM.

Asterisks represent statistical significance of the comparisons $* P<0.05$

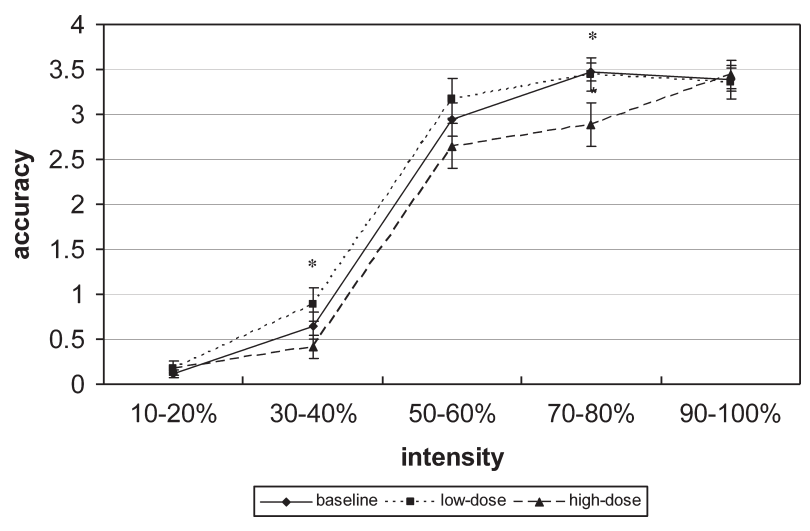

Figure 2 Fear accuracy (number of correctly recognized faces) per $20 \%$ intensity block at baseline, following low-dose depletion and high-dose depletion.

Values represent means \pm 1 SEM. Asterisks represent statistical significance of comparisons. ${ }^{*} P<0.05$

$(F(1.53,25.99)=3.9, P=0.044)$ and $70-80 \%(F(2,34)=4.5$, $P=0.018)$ intensity levels. The higher the intensity of the emotion, the better it was recognized. No difference in fear recognition between lowdose depletion and baseline was found (see Figure 2). Repeating the analysis with 13 patients, the Intervention effect was no longer significant for the $30-40 \%$ intensity level $(F(1.4,16.5)=1.6, P=0.230)$ but remained significant for the $70-80 \%$ intensity level $(F(2,24)=3.7$, $P=0.039)$. No effects of ATD on the recognition of the other emotions were found.

Speed: Analyses for the reaction times were done with Intervention and Emotion as within-subject factors. Main effects of Intervention
$(F(2,34)=7.2, P=0.002)$ and Emotion $(F(2.38,40.44)=8.4$, $P<0.001)$ revealed that ATD affected speed of recognition and that subjects were faster in recognizing certain emotions compared with others. Separate analyses per emotion showed that subjects became faster at recognizing disgust following ATD (main effect of Intervention $F(1.36,23.07)=4.0, P=0.048)$. Contrast tests showed that low-dose ATD speeded the recognition of disgust compared with baseline $(F(1,17)=10.4, P=0.005)$. High-dose also speeded the recognition of disgust compared with baseline, although this was a trend $(F(1,17)=4.2, P=0.056)$. When data were re-analysed with $N=13$, the overall Intervention effect disappeared $(F(1.3,15.9)=2.3$, $P=0.146)$, however, the difference between low-dose ATD and baseline remained significant $(F(1,12)=6.9, P=0.022)$.

The results of the other cognitive tests are shown in Table 2.

Dot-probe task: Attentional bias scores were calculated for depressive and threatening stimuli. One patient appeared to be an outlier, owing to extremely slow responses. Analyses on the attentional bias score, with Intervention as a within-subjects factor, were therefore performed with and without this patient. No effects of low-dose and high dose ATD on attentional bias were found $(P>10)$.

Implicit association test Neutral: Median latencies for congruent and incongruent word pairs were calculated (e.g., flower and positive versus. insect and positive). One outlier was found owing to a large number of incorrect responses; analyses were performed with and without this subject. Analysis with Intervention and Stimulustype (congruent versus incongruent) was performed on the reaction time data. The IAT-effect (Greenwald et al., 1998) was found, indicating that subjects were faster to respond to congruent stimuli compared with incongruent stimuli (main effect of Stimulus-type $F(1,17)=87.9, P<0.001)$. This effects was also significant for $N=13$. No effect of ATD on the IAT effect was observed.

Emotional: Two outliers were found; one made a large number of errors, the other was extremely slow to respond. Analyses were performed on median response latencies, with and without the outliers, using Intervention and Stimulus-type as within-subjects factors. Latencies for congruent and incongruent stimuli were calculated. No IAT effect was found indicating that subjects were equally fast to respond to congruent (self and positive) and incongruent (self and negative) stimuli. No effect of low-dose or high-dose depletion was found $(P>0.10)$.

Dysfunctional Attitudes Scale: DAS scores were analysed with Intervention as a within-subjects factor. No effect of low-dose or high-dose depletion on DAS scores was found.

\section{Differences between mood-responders and non-responders}

At screening, mood-responders had a higher level of depressive symptoms compared with non-responders (MADRS score $12.3 \pm$ 4.6 versus. $5.7 \pm 5.0, t(16)=3.0, P=0.009)$. Furthermore, moodresponders were better at recognizing sadness than non-responders (main effect of Mood-response $F(1,16)=6.6, P=0.021$ ). When corrected for residual depressive symptoms, the effect of Moodresponse became a trend $(F(1,15)=3.6, P=0.077)$. The 
Table 2 Cognitive tests

\begin{tabular}{|c|c|c|c|c|c|c|}
\hline & Baseline & Low-dose & High-dose & $F$ & Df & $P$ \\
\hline \multicolumn{7}{|l|}{ Dot-probe ${ }^{a}$} \\
\hline RT depressive (ms) & $514.8(70.6)$ & $519.0(97.3)$ & $526.5(81.8)$ & 0.6 & 16 & 0.569 \\
\hline RT anxious (ms) & $531.5(78.7)$ & $524.1(91.3)$ & $528.4(88.3)$ & 0.1 & 16 & 0.874 \\
\hline RT positive (ms) & $519.5(69.6)$ & $513.8(84.7)$ & $523.2(82.8)$ & 0.3 & 16 & 0.752 \\
\hline RT neutral (ms) & $535.7(87.3)$ & $524.7(90.4)$ & $533.2(84.5)$ & 0.3 & 16 & 0.727 \\
\hline$A B$ depressive- positive & $-4.7(13.3)$ & $5.2(25.7)$ & $3.3(20.7)$ & 1.0 & 16 & 0.384 \\
\hline $\mathrm{AB}$ anxious -neutral & $-4.3(13.8)$ & $-0.6(16.9)$ & $-4.8(25.5)$ & 0.3 & 16 & 0.763 \\
\hline \multicolumn{7}{|l|}{ Word Learning Task } \\
\hline Immediate recall correct ${ }^{b}$ & $47.9(11.6)$ & $47.7(9.7)$ & $43.0(11.7)$ & 4.1 & 17 & $0.026^{*}$ \\
\hline Immediate recall false & $1.0(1.3)$ & $2.0(2.2)$ & $4.3(4.8)$ & 6.0 & 17 & $0.018^{*}$ \\
\hline Delayed recall correct & $10.7(2.8)$ & $10.4(3.4)$ & $8.3(3.7)$ & 9.8 & 17 & $<0.001 * *$ \\
\hline Delayed recall false & $0.3(0.5)$ & $0.3(0.5)$ & $1.0(1.2)$ & 4.2 & 17 & $0.035^{*}$ \\
\hline \multicolumn{7}{|l|}{ Verbal Fluency } \\
\hline Number correct responses & $10.9(3.2)$ & $9.8(4.0)$ & $10.3(4.6)$ & 1.4 & 17 & 0.271 \\
\hline Number false responses & $0.5(0.8)$ & $0.3(0.6)$ & $0.3(0.6)$ & 0.3 & 17 & 0.732 \\
\hline \multicolumn{7}{|l|}{ IAT Neutral } \\
\hline RT congruent (ms) & $651.6(102.4)$ & $638.8(93.4)$ & $639.8(98.0)$ & 0.6 & 17 & 0.537 \\
\hline RT incongruent (ms) & $1005.1(226.0)$ & $998.0(213.0)$ & $1007.9(249.1)$ & 0.1 & 17 & 0.923 \\
\hline \multicolumn{7}{|l|}{ IAT Emotional } \\
\hline RT congruent (ms) & $851.3(252.0)$ & $827.1(205.0)$ & $826.1(233.7)$ & 0.8 & 17 & 0.443 \\
\hline RT incongruent (ms) & $817.7(206.9)$ & $799.9(214.0)$ & $822.0(254.8)$ & 0.4 & 17 & 0.584 \\
\hline DAS-11 & $38.9(8.8)$ & $35.7(7.6)$ & $36.5(9.4)$ & 2.9 & 34 & 0.069 \\
\hline
\end{tabular}

${ }^{\text {aD }}$ Dot-probe data are reported for $N=17$, excluding one outlier

${ }^{\mathrm{b}}$ Total of trial $1-5$

Values represent means with SD in parentheses.

$A B$, attentional bias; RT, reaction time; IAT, implicit attitudes test.

${ }^{*} P<0.05,{ }^{* *} P<0.01$. increased accuracy was not at the expense of the speed of recognition: an overall interaction effect of Emotion $\times$ Mood-response was found for speed of facial expression recognition $(F(5,80)=4.0$, $P=0.003)$ : mood-responders were faster at recognizing sadness (main effect of Mood-response $F(1,16)=4.8, P=0.043$ ) and tended to be faster at recognizing fear $(F(1,16)=3.5, P=0.080)$. However, when corrected for baseline depressive symptoms, these effects disappeared $(F(1,15)=0.9, P=0.338 ; F(1,15)=0.5$, $P=0.479$ ). A trend for an interaction effect of Intervention $\times$ Mood-response was found for the number of correct responses at immediate recall $(F(2,32)=3.2, P=0.056)$; moodresponders recalled more correct words at baseline compared with non-responders $(52.1 \pm 12.8$ versus $43.8 \pm 9.1)$ but this difference was not significant (main effect Mood-response $F(1,17)=2.5$, $P=0.132$ ). Separate analyses for mood-responders and nonresponders revealed that immediate recall performance decreased after high-dose ATD in mood-responders (main effect of Intervention $F(2,16)=6.1, P=0.011)$ but not in non-responders (main effect of Intervention $F(2,16)=0.1, P=0.911$ ). Analyses with $N=13$ revealed equal results.

\section{Effects of type of anti-depressant medication}

Twelve patients were taking an SSRI at the time of study: citalopram $20-60 \mathrm{mg}(n=5)$; paroxetine $10-40 \mathrm{mg}(n=5)$; sertraline $100 \mathrm{mg}(n=1)$; fluoxetine $40 \mathrm{mg}(n=1)$. Six patients were staking the SSNRI venlafaxine, $150-375 \mathrm{mg}$. The effect of type of medication (SSRI versus SSNRI) on the different outcome measures was investigated by including type of medication as a between-subjects factor in the repeated measures analyses. Differences were found between patients taking an SSRI and patients taking an SSNRI in that the latter group experienced more physical symptoms (main effect of Medication $F(1,15)=5.0, P=0.041)$, more depressive symptoms (main effect of Medication $F(1,16)=5.7, P=0.029$ ) and more anxiety symptoms (main effect of Medication $F(1,16)=8.5, P=0.010)$. However, these effects were independent of ATD.

A considerable overlap was found between SSNRI use and mood-response: the majority of patients taking an SSNRI were mood-responders (five out of six), compared with one third of the patients taking an SSRI (four out of twelve). 


\section{Discussion}

This study confirms that the effects of ATD on psychiatric symptoms depend on the extent of depletion. High-dose ATD led to a marked increase of symptoms, whereas low-dose ATD did not ( $91 \%$ versus $59 \%$ reduction of plasma tryptophan/LNAA ratio). Although 59\% reduction of tryptophan is thought to be sufficient to decrease brain serotonin synthesis (Biggio et al., 1974; Young et al., 1989), low-dose ATD may still be used as a placebo procedure, if the study focuses on symptoms. Furthermore, this study showed that ATD affects memory and facial expression recognition, as had previously been found in healthy subjects. Another new finding is that baseline emotional information processing, in particular the recognition of facial expressions of sadness, predicts mood-response to ATD. A number of other information processing measures have recently been found to predict depressive response to ATD: cognitive reactivity (Booij and Van der Does, 2007); emotional Stroop interference (Booij et al., 2005a) and low heart rate variability, which is associated with impaired prefrontal functioning (Booij et al., 2006).

\section{Differential effect of low-dose and high-dose ATD}

We observed few effects of low-dose ATD, since only the speed of disgust recognition was affected. While two recent studies presented stronger effects of low-dose ATD (Hayward et al., 2005; Munafò et al., 2006), the low-dose mixtures in these two studies resulted in larger decreases of tryptophan and the tryptophan/ LNAA ratio and may therefore not be comparable with our lowdose mixture (Merens and Van der Does, 2007). Furthermore, these studies compared 'low-dose' ATD to a tryptophan containing mixture, which increased tryptophan and tryptophan/LNAA ratio (by $48 \%$ and $27 \%$, respectively). This active control mixture may have increased the probability of finding an interaction effect. In the current study, low-dose ATD resulted in a $58.5 \%$ decrease of tryptophan/LNAA.

Several studies have now reported that ATD increases emotional Stroop interference (attentional bias) in healthy subjects and in recovered depressed patients (Booij et al., 2005a; Evers et al., 2006; Hayward et al., 2005; Munafò et al., 2006). However, the current study failed to find an effect of ATD on attentional bias as measured with the dot-probe test. This difference may be owing to the different tests to measure attentional bias: the dot-probe test is a measure of spatial attention, whereas performance on the Stroop test reflects several other processes. Another reason may be that we did not use the optimal stimulus presentation times. Studies using the dot-probe test have found attentional biases in depression using relatively long stimulus presentations (1 $\mathrm{s}$ or more) (Mogg et al., 1995). When stimuli are presented for shorter durations, results are mixed (Bradley et al., 1997; Mathews et al., 1996). In the current study stimuli were presented for only $500 \mathrm{~ms}$., which is the most often used procedure, but which in retrospect may explain the lack of effect on this measure. However, the dot-probe has advantages in that it circumvents the possibility of response bias interpretations, since it requires a response to a neutral stimulus (dot). Also, it allows for an investigation of both a facilitating and a possible impairing effect of the emotional stimulus on the detection of the dot, depending on the position of the emotional word relative to the dot (MacLeod et al., 1986).

\section{Specific or generalized effect of ATD on information processing}

High-dose ATD decreased the recognition of facial expressions of fear, which was not accompanied by faster or slower responses to fear. The recognition of facial expressions of disgust was speeded following both low-dose and high-dose ATD. This unexpected effect may represent a mood-congruent effect of ATD, which may be related to the unpalatable taste of the AA mixtures, but also to the self-declarative nature of depressive cognition.

The effect on fear recognition is in line with Harmer et al. (2003b) who found a similar effect in healthy females, but not in males. Since our sample included only two males, it is unclear whether this gender effect was replicated. Even though these effects are consistent, they are in contrast to the expected effects of lowered serotonin function and the symptomatic effects of ATD. Studies investigating the acute effects of other serotonin manipulations have also found effects on emotion recognition. In healthy subjects, both citalopram and tryptophan increased the recognition of fear (Attenburrow et al., 2003; Harmer et al., 2003a). However, in remitted depressed patients elevated fear recognition was normalized following citalopram administration (Bhagwagar et al., 2004). Subchronic treatment with citalopram or tryptophan decreased the recognition of negative facial expressions in healthy volunteers (Harmer et al., 2004; Murphy et al., 2006). Both the direction of the effect of ATD on facial expression recognition and the differential effects of SSRI administration on fear recognition in different samples remain to be fully explained.

In contrast with our previous findings, ATD had no effect on verbal fluency, which measures retrieval from semantic memory (Booij et al., 2005a). Effects of ATD on learning, memory retrieval and consolidation have been observed in healthy individuals (Park et al., 1994; Riedel et al., 1999; Schmitt et al., 2000). In remitted depressed patients, ATD was found to decrease memory for positive words (Booij et al., 2005b). ATD also impaired short-term memory, in recovered but not in healthy subjects and without affecting learning or long-term memory consolidation (Hayward et al., 2005). In the present study high-dose ATD impaired learning and retrieval in remitted depressed patients, which is in line with results in healthy subjects. However, the impaired learning following high-dose ATD occurred mainly in mood-responders. In contrast to previous studies, memory consolidation was not affected.

Our results give no indication for a general effect of ATD on emotional information processing. Only facial expression recognition was affected by ATD; attentional bias and implicit attitudes were unaffected. Future research should direct attention to the effects of ATD on different aspects of emotional information processing to clarify whether effects are specific for certain aspects of emotional information processing. Although the patients in the current study were repeatedly exposed to emotional stimuli, habituation to these stimuli does not seem to be an explanation for the 
absence of any effects on the IAT and dot-probe test. The observed effects of ATD on the recognition of fearful facial expression do not support this explanation. Also, we compared the effects of ATD to a baseline measure that was based on the mean of the screening and final (postintervention) session. If learning or habituation effects occur, the average of these sessions would be the best estimate of the baseline (the second and third sessions were low- and high-dose ATD in randomized order).

\section{Relationship between emotional information process- ing and serotonergic vulnerability}

Mood response to ATD was related to faster and more accurate recognition of sadness and faster recognition of fear, although when corrected for residual depressive symptoms only the effect on the accuracy of sadness recognition remained and became statistically a trend. The sample of the current study was too small to correct for clinical and demographic factors that might mediate the relationship between cognitive processing and mood response to ATD. However, the relation between recognition of sadness and mood response to ATD was not entirely related to the level of residual depressive symptoms since the effect became a trend and did not disappear when corrected for residual depressive symptoms. These results are in line with research in symptomatic and remitted depressed patients, which showed that a bias towards the perception of negative faces is a vulnerability factor for depressive relapse and that negative mood increases this negative perceptual bias (Bouhuys et al., 1999; Gur et al., 1992).

Differential results were found for SSNRI and SSRI users; however, these were mostly independent of ATD. These differences may be owing to an overlap with mood-response since five out of six SSNRI users were mood-responders.

\section{Suggestions for future research}

Facial expression recognition has been found to be related to serotonin function. However, more research on the direction of this association is needed. Since our study failed to find an effect of ATD on attentional bias, ATD studies comparing different tests to measure attentional bias may shed light on the link between serotonin and attentional bias in depression. Also, more studies on the effects of ATD on the different aspects of memory in remitted depressed patients are needed since evidence in this field mainly involves healthy subjects.

\section{Limitations}

The small sample size of the current study is a limitation. Furthermore, the relatively large number of patients who vomited following the depletion mixture warrants replication of the results in a larger sample. We carried out all analyses twice (with and without the ssfive patients who vomited in response to the AA mixture), and found only a few minor differences in results. The biochemical data, when analysed for all patients, showed a significant decrease in tryptophan levels, supporting the view that despite the vomiting, the amino-acids were well absorbed. We also compared the decreases in plasma tryptophan and tryptophan/LNAA ratio of the five patients who vomited to the patients who did not. No significant differences were observed $(P$ values $>0.08$ ); therefore we decided to include the patients who vomited in the overall analyses. Since the present study was a continuation of our previous experiments, using the same batch of AAs and the same design, procedures, recruitment and setting, it is unclear what has caused the differences in tolerability. Another limitation is the absence of a placebo condition. However, a true placebo does not exist. Studies have used mixtures containing between $2.3 \mathrm{~g}$ and $5 \mathrm{~g}$ tryptophan. This procedure leads to increases in tryptophan and the tyrptophan/LNAA ratio, which in turn may affect cognitive performance and symptoms (Markus et al., 1998; Markus et al., 2002). Including such a placebo mixture may thus lead to overestimations of the effects of ATD. A possible solution for future studies would be to use the novel ATD method of administrating a natural collagen mixture with low-tryptophan content (Lieben et al., 2004), since the placebo condition of this method seems to have no effect on tryptophan concentrations in humans (Evers et al., 2005).

\section{Acknowledgements}

The authors want to thank Adrie Seldenrijk and Julian Struijk for their assistance in data collection and the dieticians and staff of the laboratory and pharmacy of Parnassia for their contributions to this study. This study was supported by grants to A.J.W. van der Does $\mathrm{PhD}$, from the Netherlands Organization for Science- Medical Sciences (NWOMW 904-57-132) and the 'Stichting tot Steun VCVGZ'.

\section{References}

Attenburrow M J, Williams C, Odontiadis J, Reed A, Powell J, Cowen P J, Harmer C J (2003) Acute administration of nutritionally sourced tryptophan increases fear recognition. Psychopharmacology (Berl) 169: 104-107

Benkelfat C, Palmour R M, Young S N, Ellenbogen M A, Dean P (1994) Mood-lowering effect of tryptophan depletion. Enhanced susceptibility in young men at genetic risk for major affective disorders. Arch Gen Psychiatry 51: 687-697

Bhagwagar Z, Cowen P J, Goodwin G M, Harmer C J (2004) Normalization of enhanced fear recognition by acute SSRI treatment in subjects with a previous history of depression. Am J Psychiatry 161: 166-168

Biggio G, Fadda F, Fanni P, Tagliamonte A, Gessa G L (1974) Rapid depletion of serum tryptophan, brain tryptophan, serotonin and 5-hydroxyindoleacetic acid by a tryptophan-free diet. Life Sci 14: 1321-1329

Booij L, Van der Does A J W (2007) Cognitive and serotonergic vulnerability to depression: convergent findings. J Abnorm Psychol 116: 86-94

Booij L, Van der Does A J W, Haffmans P M J, Riedel W J, Fekkes D, Blom M J B (2005a) The effects of high-dose and low-dose tryptophan depletion on mood and cognitive functions of remitted depressed patients. J Psychopharmacol 19: 267-275

Booij L, Van der Does A J W, Spinhoven P, McNally R J (2005b) Acute tryptophan depletion as a model of depressive relapse. Behavioural specificity and ethical considerations. Br J Psychiatry 187: 148-154

Booij L, Swenne C A, Brosschot J F, Haffmans P M J, Thayer J F, Van der Does A J W (2006) Tryptophan depletion affects heart rate variability and impulsivity in remitted depressed patients with a history of suicidal ideation. Biol Psychiatry 60: 507-514 
Bouhuys A L, Geerts E, Gordijn M (1999) Depressed patients' perceptions of facial emotions in depressed and remitted states are associated with relapse: a longitudinal study. J Nerv Ment Dis 187: 595-602

Bradley B P, Mogg K, Lee S (1997) Attentional biases for negative information in induced and naturally occuring dysphoria. Behav Res Ther 35: 911-927

Carpenter L L, Anderson G M, Pelton G H, Gudin J A, Kirwin P D, Price L H, Heninger G R, McDougle C J (1998) Tryptophan depletion during continuous CSF sampling in healthy human subjects. Neuropsychopharmacol 19: $26-35$

De Jong P J (2002) Implicit self-esteem and social anxiety: differential selffavouring effects in high and low anxious individuals. Behav Res Ther 40: $501-508$

Delgado P L, Charney D S, Price L H, Aghajanian G K, Landis H, Heninger G R (1990) Serotonin function and the mechanism of antidepressant action. Arch Gen Psychiatry 47: 411-418

Egloff B, Schmukle S (2002) Predictive validity of an Implicit Association Test for assessing anxiety. J Pers Soc Psychol 83: 1441-1455

Ekman P, Friesen W (1976) Pictures of facial affect [slides]. Consulting Psychologists Press, Palo Alto, CA, USA

Evers E A T, Tillie D E, van der Veen F M, Lieben C K, Jolles J, Deutz N E P, Schmitt J A J (2005) Effects of a novel method of acute tryptophan depletion on plasma tryptophan and cognitive performance in healthy volunteers. Psychopharmacology (Berl) 178: 92

Evers E A T, van der Veen F M, Jolles J, Deutz N E P, Schmitt J A J (2006) Acute tryptophan depletion improves performance and modulates the BOLD response during a Stroop task in healthy females. NeuroImage 32: 248-255

Fekkes D, van Dalen A, Edelman M, Voskuilen A (1995) Validation of the determination of amino acids in plasma by high-performance liquid chromatography using automated pre-column derivatization with o-phthaldialdehyde. J Chromatogr B Biomed Appl 669: 177-186

Field A (2005) Discovering statistics using SPSS. Sage Publications, London, UK

First M, Spitzer R, Gibbon M, Williams J (1995) Structured Clinical Interview for DSM-IV Axis I Disorders. Patient edition (SCID-I/P). Biometrics Research Department, NYSPI, New York

Greenwald A G, Banaji M R (1995) Implicit social cognition - attitudes, self-esteem, and stereotypes. Psychol Rev 102: 4-27

Greenwald A G, McGhee D E, Schwartz J L (1998) Measuring individual differences in implicit cognition: the implicit association test. J Pers Soc Psychol 74: 1464-1480

Gur R C, Erwin R J, Gur R E, Zwil A S, Heimberg C, Kraemer H C (1992) Facial emotion discrimination: II. Behavioral findings in depression. Psychiatry Res 42: 241-251

Harmer C J, Bhagwagar Z, Perrett D I, Völlm B A, Cowen P J, Goodwin G M (2003a) Acute SSRI administration affects the processing of social cues in healthy volunteers. Neuropsychopharmacol 28: 148-152

Harmer C J, Rogers R, Tunbridge E, Cowen P J, Goodwin G (2003b) Tryptophan depletion decreases the recognition of fear in female volunteers. Psychopharmacology (Berl) 167: 411-417

Harmer C J, Shelley N, Cowen P J, Goodwin G M (2004) Increased positive versus negative affective perception and memory in healthy volunteers following selective serotonin and norepinephrine reuptake inhibition. Am J Psychiatry 161: 1256-1263

Hayward G, Goodwin G, Cowen P J, Harmer C J (2005) Low-dose tryptophan depletion in recovered depressed patients induces changes in cognitive processing without depressive symptoms. Biol Psychiatry 57: 517-524

Klaassen T, Riedel W J, Deutz N E P, van Praag H M (2002) Mood congruent memory bias induced by tryptophan depletion. Psychol Med 32: 167-172

Krahn L, Lu P, Klee G, Delgado P, Lin S, Zimmermann R (1996) Examining serotonin function: a modified technique for rapid tryptophan depletion. Neuropsychopharmacol 15: 325-328
Lavy E, van den Hout M, Arntz A (1993) Attentional bias and spider phobia: conceptual and clinical issues. Behav Res Ther 31: 17-24

Lieben C K J, Blokland A, Westerink B, Deutz N E P (2004) Acute tryptophan and serotonin depletion using an optimized tryptophan-free proteincarbohydrate mixture in the adult rat. Neurochem Int 44: 9-16

MacLeod C, Mathews A, Tata P (1986) Attentional bias in emotional disorders. J Abnorm Psychol 95: 15-20

Markus C R, Olivier B, de Haan E H F (2002) Whey protein rich in alphalactalbumin increases the ratio of plasma tryptophan to the sum of the other large neutral amino acids and improves cognitive performance in stress-vulnerable subjects. Am J Clin Nutr 75: 1051-1056

Markus C R, Panhuysen G, Tuiten A, Koppeschaar H, Fekkes D, Peters M L (1998) Does carbohydrate-rich, protein-poor food prevent a deterioration of mood and cognitive performance of stress-prone subjects when subjected to a stressful task? Appetite 31: 49-65

Mathews A, Ridgeway V, Williamson D A (1996) Evidence for attention to threatening stimuli in depression. Behav Res Ther 34: 695-705

McCabe S B, Gotlib I H, Martin R A (2000) Cognitive vulnerability for depression: Deployment of attention as a function of history of depression and current mood state. Cogn Ther Res 24: 427-444

Merens, W, Van der Does, A J W (2007) Low-dose tryptophan depletion. Biol Psychiatry 62: 542-543

Mogg K, Bradley B P, Williams R (1995) Attentional bias in anxiety and depression. The role of awareness. Br J Clin Psychol 34: 17-36

Montgomery S, Asberg M (1979) A new depression scale designed to be sensitive to change. Br J Psychiatry 134: 382-389

Munafò M R, Harmer C J, Hayward G (2006) Selective processing of social threat cues following acute tryptophan depletion. J Psychopharmacol 20: $33-39$

Murphy S E, Longhitano C, Ayres R, Cowen P J, Harmer C J (2006) Tryptophan supplementation induces a positive bias in the processing of emotional material in healthy female volunteers. Psychopharmacology (Berl) 187: 121-130

Nishizawa S, Benkelfat C, Young S N, Leyton M, Mzengeza S, de Montigny C, Blier P, Diksic M (1997) Differences between males and females in rates of serotonin synthesis in human brain. Proc Natl Acad Sci USA 94: 5308-5313

Park S B, Coull J T, McShane R H, Young A H, Sahakian B J, Robbins T W, Cowen P J (1994) Tryptophan depletion in normal volunteers produces selective impairments in learning and memory. Neuropharmacology 33: 575-588

Riedel W J, van Someren A, van Praag H M, Klaassen T, Deutz N E P (1999) Tryptophan depletion in normal volunteers produces selective impairment in memory consolidation. Psychopharmacology (Berl) 141: 362-369

Saan R, Deelman B (1986) De 15-woordentest A en B (een voorlopige handleiding). Afdeling Neuropsychologie, AZG, Groningen

Schmitt J A J, Jorissen B, Sobczak S, Van Boxtel M, Hogervorst E, Deutz N E P, Riedel W J (2000) Tryptophan depletion impairs memory consolidation but improves focussed attention in healthy young volunteers. J Psychopharmacol 14: 21-29

Tyrer P, Owen R T, Cicchetti D V (1984) The Brief Scale for Anxiety - A subdivision of the Comprehensive Psychopathological Rating-Scale. J Neurol Neurosurg Psychiatry 47: 970-975

Van der Does A J W (2001) The effects of tryptophan depletion on mood and psychiatric symptoms. J Affect Disord 64: 107-119

Weissman A (1979) The Dysfunctional Attitude Scale: A validation study. Dissertation Abstracts International 40: 1389-1390

Young S N, Ervin F R, Pihl R O, Finn P (1989) Biochemical aspects of tryptophan depletion in primates. Psychopharmacology (Berl) 98: 508-511

Young S N, Smith S E, Pihl R O, Ervin F R (1985) Tryptophan depletion causes a rapid lowering of mood in normal males. Psychopharmacology (Berl) 87: 173-177 